The Astrophysical Journal, 530:L45-L48, 2000 February 10

(C) 2000. The American Astronomical Society. All rights reserved. Printed in U.S.A.

\title{
EVOLUTIONARY MODEL AND OSCILLATION FREQUENCIES FOR $\alpha$ URSAE MAJORIS: A COMPARISON WITH OBSERVATIONS
}

\author{
D. B. Guenther, ${ }^{1}$ P. Demarque,${ }^{2}$ D. Buzasi,${ }^{3}$ J. Catanzarite,${ }^{4}$ R. Laher,${ }^{4}$ T. Conrow,${ }^{4}$ and T. Kreidl ${ }^{5}$ \\ Received 1999 August 4; accepted 1999 December 15; published 2000 January 11
}

\begin{abstract}
Inspired by the observations of low-amplitude oscillations of $\alpha$ Ursae Majoris A by Buzasi et al. using the WIRE satellite, a grid of stellar evolutionary tracks has been constructed to derive physically consistent interior models for the nearby red giant. The pulsation properties of these models were then calculated and compared with the observations. It is found that, by adopting the correct metallicity and for a normal helium abundance, only models in the mass range of 4.0-4.5 $M_{\odot}$ fall within the observational error box for $\alpha$ UMa A. This mass range is compatible, within the uncertainties, with the mass derived from the astrometric mass function. Analysis of the pulsation spectra of the models indicates that the observed $\alpha \mathrm{UMa}$ oscillations can be most simply interpreted as radial (i.e., $l=0) p$-mode oscillations of low radial order $n$. The lowest frequencies observed by Buzasi et al. are compatible, within the observational errors, with model frequencies of radial orders $n=0,1$, and 2 for models in the mass range of 4.0-4.5 $M_{\odot}$. The higher frequencies observed can also be tentatively interpreted as higher $n$-valued radial $p$-modes, if we allow that some $n$-values are not presently observed. The theoretical $l=1,2$, and 3 modes in the observed frequency range are $g$-modes with a mixed mode character, that is, with $p$-mode-like characteristics near the surface and $g$-mode-like characteristics in the interior. The calculated radial $p$-mode frequencies are nearly equally spaced, separated by $2-3 \mu \mathrm{Hz}$. The nonradial modes are very densely packed throughout the observed frequency range and, even if excited to significant amplitudes at the surface, are unlikely to be resolved by the present observations.
\end{abstract}

Subject headings: stars: evolution — stars: individual ( $\alpha$ Ursae Majoris A) — stars: oscillations

\section{INTRODUCTION}

Recently, Buzasi et al. (1999), using the star camera on the failed WIRE satellite to observe the bright star $\alpha$ Ursae Majoris A, have succeeded in detecting discrete periodic variations in luminosity with maximum amplitudes of $300 \mu \mathrm{mag}$ and oscillation frequencies between 1 and $30 \mu \mathrm{Hz}$. Oscillations that could be of a similar nature, but with amplitudes that are an order of magnitude larger and observed with lower frequency resolution, had been previously discovered among more luminous red giant stars in the globular cluster 47 Tuc, using the Hubble Space Telescope (Edmonds \& Gilliland 1996). The nature of the 47 Tuc giant oscillations remains uncertain because of the sparseness of the data. The discovery of identifiable low-amplitude oscillations in $\alpha$ Ursae Majoris now holds the promise that we may be able to apply, for the first time, the techniques of seismology to probe the interior of a red giant star.

Our purpose, in this Letter, is to describe the stellar evolutionary tracks and the interior models that we have constructed for $\alpha$ UMa A. We emphasize that these models are not empirical "fits" to the observed frequencies. They are constructed independently from the pulsation information and are based only on other available fundamental data about the $\alpha$ UMa system, i.e., the metallicity, the effective temperature, and the parallax.

\footnotetext{
${ }^{1}$ Department of Astronomy and Physics, Saint Mary's University, Halifax, NS B3H 3C3, Canada; guenther@ap.stmarys.ca.

${ }^{2}$ Center for Solar and Space Research, Department of Astronomy, Yale University, P.O. Box 208101, New Haven, CT 06520-8101; demarque@astro yale.edu.

${ }^{3}$ Space Sciences Laboratory, University of California, Berkeley, Berkeley, CA 94720; dbuzasi@ssl.berkeley.edu.

${ }^{4}$ Infrared Processing and Analysis Center, MS 100-22, California Institute of Technology, Pasadena, CA 91125; jhc@ipac.caltec.edu, laher@ipac.caltech .edu, tim@ipac.caltech.edu.

${ }^{5}$ Department of Physics and Astronomy, Northern Arizona University, Box 6010, Flagstaff, AZ 86011-6010; tobias.kreidl@ nau.edu.
}

Our aim is simply to compare the calculated oscillation mode frequencies of state-of-the-art stellar models with the observed frequencies and to draw preliminary conclusions as to the nature of the oscillations and the internal structure of $\alpha$ UMa A.

\section{EVOLUTIONARY MODELS}

\subsection{Choice of Parameters}

The primary star $\alpha$ UMa A (spectral type K0 III) is in a well-studied visual binary system. The combined mass of the system is $5.94 \pm 0.48 M_{\odot}$ (Söderhjelm 1999), derived from astrometric measurements. The secondary is believed to be a dwarf, most often classified as F7 V, although ultraviolet observations have suggested a late-A spectral type (Kondo, Morgan, \& Modisette 1977; Ayres, Marstad, \& Linsky 1981). It follows that its mass must then be between 1 and $2 M_{\odot}$ and that the primary must have a mass between 4 and $5 M_{\odot}$. This implies an age between 100 and 200 Myr. The spectral range of the secondary, corresponding to masses in the range of 1.3-1.8 $M_{\odot}$, further constrains the primary mass to the range of $4.1-4.6 M_{\odot}$.

We have explored evolutionary models in the mass range of 4.0-5.0 $M_{\odot}$ but could only achieve satisfactory agreement in the $\mathrm{H}-\mathrm{R}$ diagram in the mass range of $4.0-4.5 M_{\odot}$, as illustrated in Figure 1. This would suggest that the secondary is as massive as 1.6 or $1.7 M_{\odot}$, which is compatible with a late-A or early$\mathrm{F}$ spectral type. It may be that, owing to its faintness relative to the primary, the secondary has been assigned too late a spectral type or that, alternatively, also owing to the large brightness difference between the two stars, the orbital parameters may be slightly in error, and the combined mass was overestimated.

In constructing the models, a luminosity of $\log \left(L / L_{\odot}\right)=$ 2.5 was adopted for $\alpha \mathrm{UMa} \mathrm{A}$. The total uncertainty in $\log \left(L / L_{\odot}\right)$ was taken to be \pm 0.05 . This uncertainty combines 


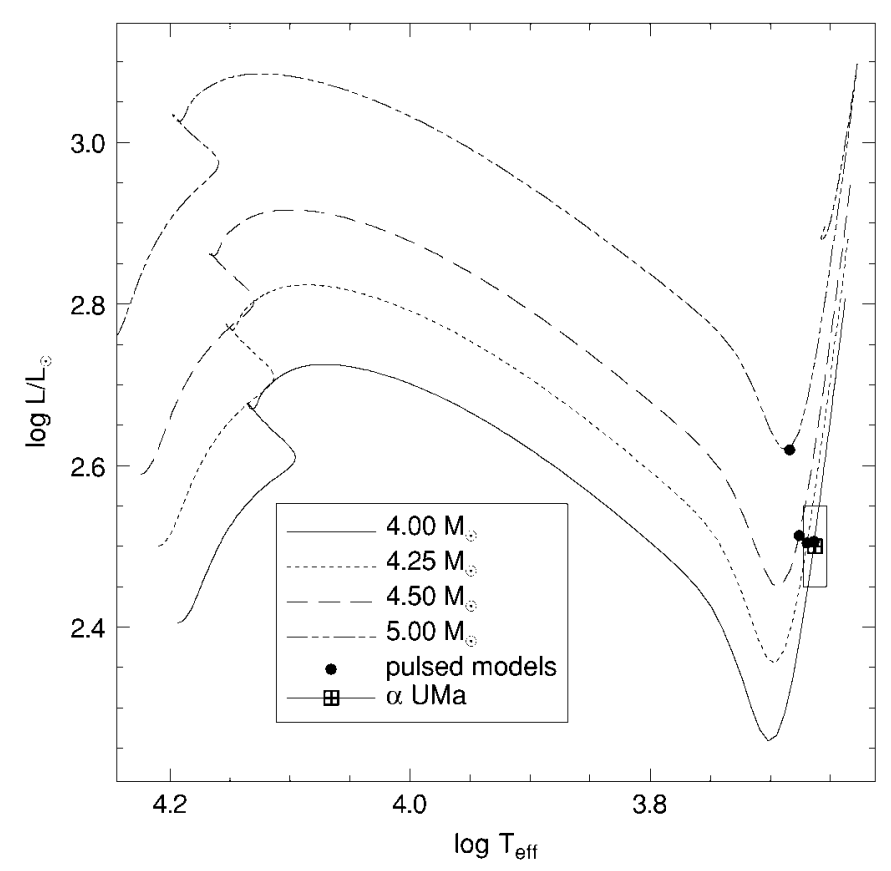

FIG. 1.-H-R diagram showing evolutionary tracks from the zero-age main sequence to the point of helium ignition on the giant branch for stellar models with masses of 4.00, 4.25, 4.50, and $5.00 M_{\odot}$. The models used in the pulsation analysis (pulsed models), whose characteristics are listed in Table 1, are indicated by data points. The three lower mass models most closely match the position of $\alpha \mathrm{UMa}$ in the $\mathrm{H}-\mathrm{R}$ diagram, within the errors. The $5 M_{\odot}$ model falls outside the error box.

the uncertainties in the parallax, the bolometric correction for $\alpha$ UMa A, and the absolute magnitude of the Sun. The luminosity is consistent with the Hipparcos parallax (Perryman 1997) and the parallax given by Söderhjelm (1999). We adopted the effective temperature $T_{\text {eff }}=4660 \mathrm{~K}$ quoted by Taylor (1999) from recent spectroscopic studies. Although Taylor's error estimate is $\pm 25 \mathrm{~K}$, we used $\pm 100 \mathrm{~K}$. As the surface gravity and radius of $\alpha \mathrm{UMa}$ are also known, we have two additional consistency checks for our models: (1) the model radius must be $\approx 25 R_{\odot}$ (Bell 1993) — this condition is automatically satisfied by the luminosity and effective temperature requirements, and (2) the surface gravity of the models must be consistent with the spectroscopically derived surface gravity, $\log g=2.03-2.23$ (Cayrel de Strobel et al. 1992) - this requirement is satisfied by the choice of mass of the models (see Table 1).

The metallicity of $\alpha$ UMa was derived spectroscopically by Taylor (1999) to be $[\mathrm{Fe} / \mathrm{H}]=-0.19$. For the helium abundance, which cannot be measured directly spectroscopically, we assumed that the Galactic helium enrichment is given by $\Delta Y / \Delta Z=2.5$. Adopting $Y_{0}=0.23$ and $Z_{0}=0.0$ for the primordial galactic abundances and taking $Z_{\odot}=0.0188$, we de- rived the composition for $\alpha$ UMa to be $X=0.727, Y=$ 0.261 , and $Z=0.0124$ (this yields $[\mathrm{Fe} / \mathrm{H}]=-0.18$ ).

\subsection{Stellar Models}

The evolutionary sequences were calculated using the nonrotating version of the Yale Stellar Evolution Code (see Guenther \& Demarque 1997). The models included the OPAL opacities in the interior (Rogers \& Iglesias 1994) and the lowtemperature opacities from Alexander \& Ferguson (1994). The OPAL equation of state (Iglesias \& Rogers 1996) was used. Diffusion of helium and the heavy elements was ignored. Most of the diffusion effects, sometimes important near the main sequence, would have been mostly obliterated by dredge-up in the deep convection zone on the giant branch and are at any rate second-order refinements in the context of the present study. For the atmospheric boundary condition, we used the Eddington approximation in the optically thin layers. A mixing length-to-pressure scale height ratio of 1.6 was used in the superadiabatic layer of the convection zone.

The most relevant evolutionary tracks, beginning on the zero-age main sequence and ending on the giant branch at the point of ignition of helium burning, for masses 4.0, 4.25, and 4.5 $M_{\odot}$, and slightly beyond the point of helium ignition, for 5.0 $M_{\odot}$, are shown in the theoretical H-R diagram in Figure 1. For each track, a model that lies closest to the position of $\alpha \mathrm{UMa}$ in the H-R diagram was selected (indicated by a data point in Fig. 1) for pulsation analysis. Table 1 lists the details of the selected models. From left to right, the table lists the mass in units of solar mass, $\log \left(L / L_{\odot}\right), \log T_{\text {eff }}$, the age in gigayears, the mass of the convective envelope in solar units, the position of the base of the convective envelope in radius fraction, and the logarithm of the pressure, temperature, density, and surface gravity (all in cgs units).

\section{PULSATION ANALYSIS}

\subsection{Overview}

We calculated the low- $l$ pulsation spectra of our models over the observed frequency range using Guenther's nonadiabatic, nonradial pulsation program (Guenther 1994). Very little analysis of the pulsation properties of stars of the mass and evolutionary phase of $\alpha \mathrm{UMa}$ has been published. Shibahashi \& Osaki (1976) included a $5 M_{\odot}$ star in their study of the excitation of $g$-modes in hydrogen shell-burning stars, finding that all the $g$-modes in their $5 M_{\odot}$ red giant model are stable.

Because $\alpha \mathrm{UMa}$ is a moderately luminous red giant star ascending the giant branch for the first time, its structure is characterized by a burnt out helium core, a hydrogen-burning shell, and a deep convective envelope. In Figure 2, we show the square of the Brunt-Väisälä frequency $N^{2}$ (in units of $\mathrm{s}^{-2}$ ) and the square of the $l=1 \mathrm{Lamb}$ frequency $L_{l}^{2}$ (in units of $\mathrm{s}^{-2}$ ) plotted as a function of the radius fraction for our $4.25 M_{\odot}$

TABLE 1

$\alpha$ Ursae Majoris A Model Characteristics

\begin{tabular}{cccccccccc}
\hline \hline $\begin{array}{c}\text { Mass } \\
\left(M_{\odot}\right)\end{array}$ & $\log \left(L / L_{\odot}\right)$ & $\log T_{\text {eff }}$ & $\begin{array}{c}\text { Age } \\
(\mathrm{Gyr})\end{array}$ & $M_{\text {env }}$ & $x_{\text {env }}$ & $\begin{array}{c}\log P_{c} \\
(\mathrm{cgs})\end{array}$ & $\begin{array}{c}\log T_{c} \\
(\mathrm{cgs})\end{array}$ & $\begin{array}{c}\log \rho_{c} \\
(\mathrm{cgs})\end{array}$ & $\begin{array}{c}\log g \\
(\mathrm{cgs})\end{array}$ \\
\hline $4.00 \ldots \ldots$ & 2.5064 & 3.6633 & 0.1408 & 2.3614 & 0.3150 & 19.9891 & 7.9306 & 4.2105 & 2.1391 \\
$4.25 \ldots \ldots$ & 2.5043 & 3.6692 & 0.1213 & 1.7861 & 0.4170 & 19.8900 & 7.9239 & 4.1263 & 2.1912 \\
$4.50 \ldots \ldots$ & 2.5137 & 3.6756 & 0.1058 & 1.2030 & 0.5006 & 19.8349 & 7.9263 & 4.0742 & 2.2321 \\
$5.00 \ldots \ldots$ & 2.6197 & 3.6839 & 0.0832 & 0.5137 & 0.5978 & 19.7780 & 7.9414 & 4.0095 & 2.2049 \\
\hline
\end{tabular}




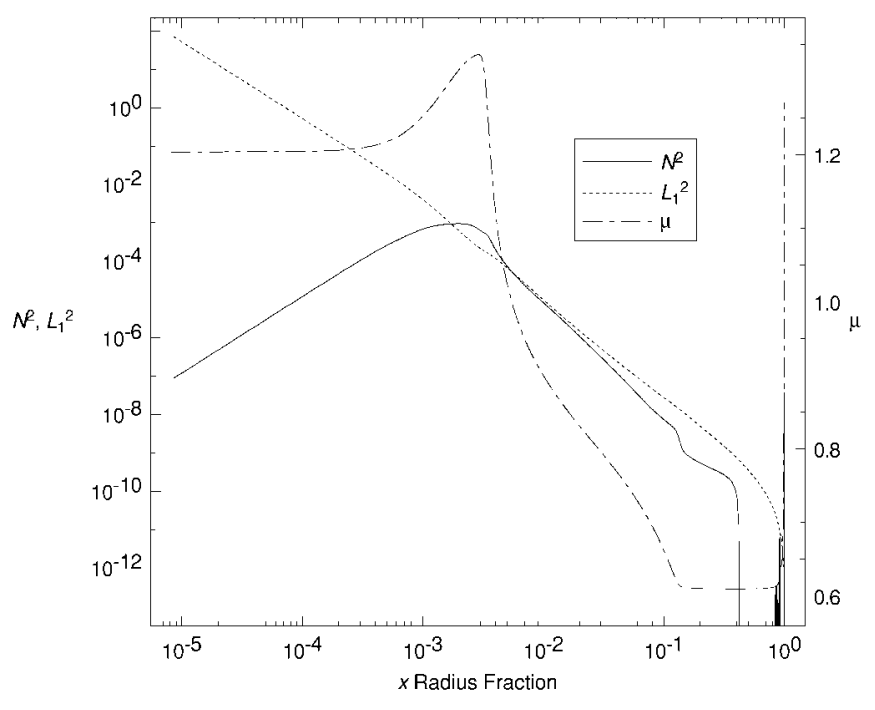

FIG. 2.-The square of the Brunt-Väisälä frequency $N^{2}$ and the square of the $l=1$ Lamb frequency $L_{l}^{2}$ plotted as a function of the radius fraction for our $4.25 M_{\odot}$ model, with the squared frequency scale (in units of $\mathrm{s}^{-2}$ ) indicated on the left $y$-axis. Also plotted as a function of the radius fraction is the mean molecular weight $\mu$, with the scale indicated on the right $y$-axis.

model. This propagation diagram conveniently illustrates the regions in the stellar interior where $g$-modes and $p$-modes can propagate, since $g$-modes exist only in regions where their frequency is less than both the Lamb frequency and the BruntVäisälä frequency, and similarly $p$-modes exist where their frequency is greater than both the Lamb and Brunt-Väisälä frequencies (Scuflaire 1974; see also Unno et al. 1989).

For $\alpha \mathrm{UMa}$, we see that there is an inner region where $g$ -

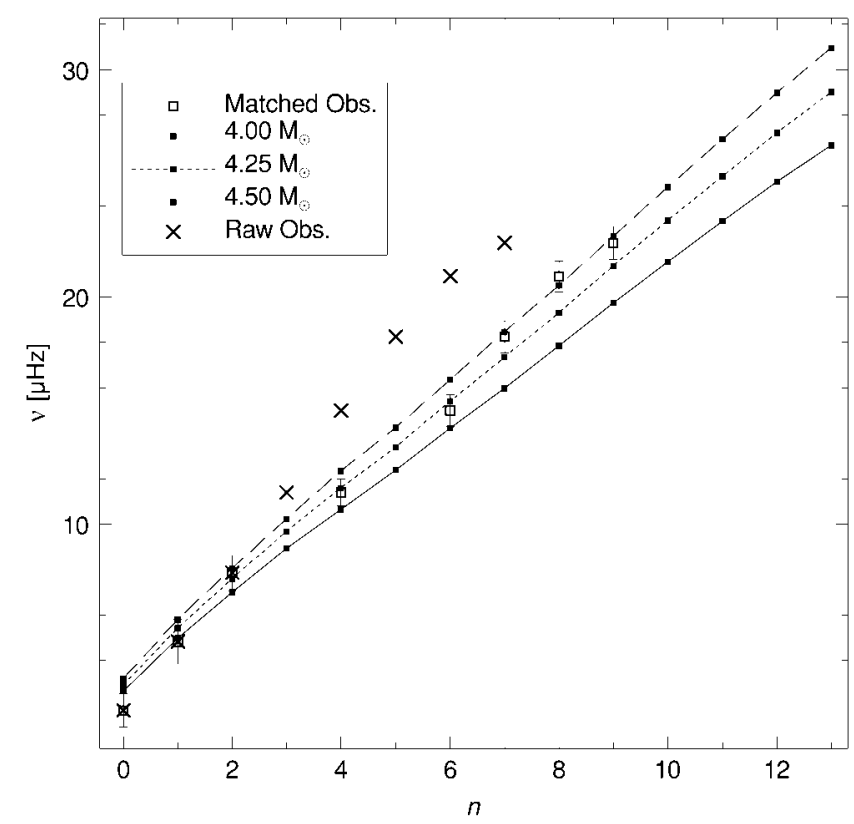

FIG. 3.-The $l=0 p$-modes for the $4.00,4.25$, and $4.50 M_{\odot}$ models of $\alpha$ UMa plotted as a function of their radial order $n$. The observed oscillation frequencies are also plotted using two different $n$-value-matching schemes. The frequencies labeled "Raw Obs." are plotted sequentially starting from $n=0$, and for the frequencies labeled "Matched Obs.," the $n$-value identification was chosen to fit the predicted mode data of the models.
TABLE 2

Radial $p$-Mode Frequencies of $\alpha$ UMa Models ${ }^{\mathrm{a}}$

\begin{tabular}{lrrrr}
\hline \hline \multicolumn{1}{c}{$n$} & $4.00 M_{\odot}$ & $4.25 M_{\odot}$ & $4.50 M_{\odot}$ & $5.00 M_{\odot}$ \\
\hline $0 \ldots \ldots$ & 2.67 & 2.94 & 3.22 & 3.27 \\
$1 \ldots \ldots$. & 5.01 & 5.44 & 5.82 & 5.54 \\
$2 \ldots \ldots$. & 7.00 & 7.60 & 8.07 & 7.51 \\
$3 \ldots \ldots$. & 8.94 & 9.68 & 10.25 & 9.48 \\
$4 \ldots \ldots$. & 10.65 & 11.59 & 12.34 & 11.50 \\
$5 \ldots \ldots$. & 12.38 & 13.40 & 14.25 & 13.33 \\
$6 \ldots \ldots$. & 14.24 & 15.42 & 16.36 & 15.24 \\
$7 \ldots \ldots$. & 16.00 & 17.35 & 18.48 & 17.29 \\
$8 \ldots \ldots$. & 17.85 & 19.31 & 20.52 & 19.26 \\
$9 \ldots \ldots$. & 19.73 & 21.36 & 22.67 & 21.23 \\
$10 \ldots \ldots$ & 21.55 & 23.36 & 24.84 & 23.28 \\
$11 \ldots \ldots$. & 23.33 & 25.31 & 26.94 & 25.30 \\
$12 \ldots \ldots$ & 25.07 & 27.22 & 28.97 & 27.21 \\
\hline
\end{tabular}

${ }^{\mathrm{a}}$ Frequencies are in units of microhertz.

modes can propagate, i.e., where $\omega^{2} \leq N^{2}$ and $\omega^{2} \leq L_{l}^{2}$, and an outer region, near the surface, where the $g$-modes are damped. The $g$-modes are effectively trapped by the gradient in the mean molecular weight $\mu$ (see the plot of $\mu$ vs. the radius fraction in Fig. 2) left by the retreating convective envelope. The BruntVäisälä frequency corresponds to the natural buoyancy frequency of the medium, which is the frequency at which a fluid element would bob up and down if displaced slightly from its equilibrium depth. Steep changes in the mean molecular weight enhance the buoyancy-restoring force, which in turn affects the Brunt-Väisälä frequency. As a consequence, the Brunt-Väisälä frequency for $\alpha \mathrm{UMa}$ has a relatively high peak in the interior (the trapezoidal shape of the Brunt-Väisälä frequency curve for the Sun is not as sharply peaked). This allows for the existence of $g$-modes at high frequencies that overlap the $p$-mode frequency spectrum. Indeed, within the observed frequency range, $\omega^{2} \geq 1.31 \times 10^{-10} \mathrm{~s}^{-2}$, we find that all the $l=1,2$, and 3 modes are high $n$-valued $g$-modes with $p$-mode character in the outer envelope and $g$-mode character in the interior; i.e., they are mixed modes. ${ }^{6}$ Within the standard nomenclature system, these modes are classified as $g$-modes because the radial order of the $g$-mode part, $n_{g}$, of the eigenfunction greatly exceeds the radial order of the $p$-mode part, $n_{p}$.

\subsection{Comparison with Observations}

A first comparison of the observed frequencies and the magnitudes of their spacings with calculated mode frequencies does suggest that the observed modes are low $n$-valued radial $p$ modes. Indeed, the three lowest observed frequencies correspond, within the errors, to the $(l=0) p$-modes frequencies for $n=1,2$, and 3, respectively (see Fig. 3). The nonradial modes (all $g$-modes) are very densely packed within the observed frequency range, with separations on the order of $0.1 \mu \mathrm{Hz}$; hence, even if excited at the surface to appreciable amplitudes, they are not resolvable with the current observations.

In Figure 3, we plot frequency versus $n$ for the $l=0$ $p$-modes of our $4.00,4.25$, and $4.50 M_{\odot}$ models. We have not plotted the frequencies of the $5.0 M_{\odot}$ model because they lie almost exactly on top of the frequencies of the $4.25 M_{\odot}$ model. The radial $p$-mode frequencies for all the models are listed in Table 2. Figure 3 also contains two different representations

\footnotetext{
${ }^{6}$ Mixed modes, characterized by mode bumping, may have been observed in $\eta$ Bootis (Kjeldsen et al. 1995; Christensen-Dalsgaard, Bedding, \& Kjeldsen 1995; Guenther \& Demarque 1996).
} 
of the observed modes for comparison. If we try to match the observed frequencies with the closest model frequencies, we find that the first three observed modes match well the $n=0$, 1 , and 2 model frequencies. The fourth observed mode matches the $n=4$ model mode, and the fifth through eighth observed modes match the $n=6,7,8$, and 9 model modes. This tentative identification of the observations with the model predictions is represented by the data points labeled "Matched Obs." We could have also assumed that the next odd $l$-valued $p$-mode $(n=7)$ is also missing from the observations, creating an even better fit for the next three $p$-modes. The data points labeled "Raw Obs." simply plot the observational data in sequence, beginning with $n=0$.

Regardless of whether or not we try to match all the observed frequencies, we do find that the fundamental and first two harmonics of the radial $p$-modes of our models (i.e., models that have the mass, chemical composition, and observed H-R diagram position of $\alpha \mathrm{UMa} \mathrm{A}$ ) are compatible with the observations of Buzasi et al. (1999). Many questions remain unanswered regarding the higher $n$-valued radial $p$-modes. It may be that the $n=3$ and $n=5$, and possibly $n=7, p$-modes are not being excited to large enough amplitudes to be observed or that the structure of the outer envelope of our model, which is determined by the mixing-length approximation, is the cause of the discrepancy (see, e.g., Demarque, Guenther, \& Kim 1997 for the case of the Sun). Alternatively, if we discount the possibility of gaps in the continuum of observed modes, then the implication is that the mass of $\alpha \mathrm{UMa} \mathrm{A}$ is significantly greater than $4.5 M_{\odot}$. In this case, the fact that our models diverge from the data would indicate that there are fundamental problems with current theories of stellar structure on the giant branch. However, a larger mass would be in disagreement with the dynamical mass, the evolutionary mass, and the spectroscopic estimate of $\log g$ for $\alpha \mathrm{UMa}$, and we therefore view this possibility as unlikely.

\subsection{The Pulsation Mass of $\alpha U M a A$}

If we accept the observations and the matching of the three lowest $n$-valued modes, then the three lowest observed frequencies place a strong constraint on the mass of $\alpha \mathrm{UMa} \mathrm{A}$, which can be seen to be $4.25 \pm 0.25 M_{\odot}$. A more precise error estimate would be difficult, in view of the uncertainties in the input parameters of the models, such as chemical composition, luminosity, and effective temperature. Since the principal uncertainties in our models are found in the outer convective layers, we do expect better agreement with observations using the low- $n$ radial $p$-modes.

\section{SUMMARY}

In order to analyze the low-amplitude variability observed in $\alpha$ UMa by Buzasi et al. (1999), using the WIRE satellite, we have constructed stellar evolutionary models for $\alpha \mathrm{UMa} \mathrm{A}$ and evaluated their oscillation frequencies. Our main findings are the following:

1. The three lowest frequencies observed in $\alpha$ UMa are most simply interpreted as radial $p$-modes $(l=0)$ with radial order $n=0,1$ and 2 .

2. This interpretation is compatible with a derived pulsation mass of $4.25 \pm 0.25 M_{\odot}$, in good agreement with the mass inferred from the known astrometric mass function.

3. We can tentatively match all of the observed frequencies to model frequencies if we assume that the observations do not see the $n=3$ and $n=5$, and possibly the $n=7$, radial $p$ modes. We note that the large error bars on the observations, and the uncertainties in the parameters of the stellar models, allow one to invent a variety of different matchings; hence, one cannot yet fit models to the $p$-mode observations. Taking the extreme point of view of assuming that the internal structure of $\alpha \mathrm{UMa} \mathrm{A}$ is Sun-like, and using the observed spacings, leads to the conclusion that $\alpha$ UMa A has a mass near $10 M_{\odot}$, a result incompatible with the evidence from the binary orbit, stellar evolution, and the spectroscopic estimate of $\log g$.

4. The low- $l$ nonradial modes in the observed frequency range are all $g$-modes; more specifically, they are mixed modes. Because they are damped through the convective envelope, they are unlikely to have significant amplitudes at the surface. At any rate, they are spaced so closely (by approximately $0.1 \mu \mathrm{Hz}$ ) for a given $l$ that they would not be resolvable with the current observations.

This work was supported in part by an NSERC grant to D. B. G. and NASA grant NAG5-8406.

\section{REFERENCES}

Alexander, D. R., \& Ferguson, J. W. 1994, ApJ, 437, 879

Ayres, T. R., Marstad, N. C., \& Linsky, J. L. 1981, ApJ, 247, 545

Bell, R. A. 1993, MNRAS, 264, 345

Buzasi, D., et al. 1999, preprint

Cayrel de Strobel, G., Hauck, B., François, P., Thévenin, F., Friel, E., Mermilliod, M., \& Borde, S. 1992, A\&AS, 95, 273

Christensen-Dalsgaard, J., Bedding, T. R., \& Kjeldsen, H. 1995, ApJ, 443, L29

Demarque, P., Guenther, D. B., \& Kim, Y.-C. 1997, ApJ, 474, 790

Edmonds, P. D., \& Gilliland, R. L. 1996, ApJ, 464, L157

Guenther, D. B. 1994, ApJ, 422, 400

Guenther, D. B., \& Demarque, P. 1996, ApJ, 456, 798

. 1997, ApJ, 484, 937
Iglesias, C. A., \& Rogers, F. J. 1996, ApJ, 464, 943

Kjeldsen, H., Bedding, T. R., Viskum, M., \& Frandsen, S. 1995, AJ, 109, 1313

Kondo, Y., Morgan, T. H., \& Modisette, J. L. 1977, PASP, 89, 675

Perryman, M. A. C., ed. 1997, The Hipparcos and Tycho Catalogues (ESA SP-1200; Noordwijk: ESA)

Rogers, F. J., \& Iglesias, C. A. 1994, Science, 263, 50

Scuflaire, R. 1974, A\&A, 36, 107

Shibahashi, H., \& Osaki, Y. 1976, PASJ, 28, 533

Söderhjelm, S. 1999, A\&A, 341, 121

Taylor, B. J. 1999, A\&AS, 134, 523

Unno, W., Osaki, Y., Ando, H., Saio, H., \& Shibahashi, H. 1989, Nonradial Oscillations of Stars (2d ed.; Tokyo: Univ. Tokyo Press) 